\title{
Epidemiological study of high-risk human papillomavirus infection in subjects with abnormal cytological findings in cervical cancer screening
}

\author{
WEIZHI YOU ${ }^{1}$, SHAOCONG LI $^{1}$, RAN DU $^{2}$, JIZENG ZHENG ${ }^{3}$ and AIFANG SHEN ${ }^{1}$ \\ Departments of ${ }^{1}$ Obstetrics and Gynecology and ${ }^{2}$ Pathology, Liaocheng People's Hospital; \\ ${ }^{3}$ Department of Obstetrics and Gynecology, Liaocheng Third People's Hospital, Liaocheng, Shandong 252000, P.R. China
}

Received July 13, 2016; Accepted May 5, 2017

DOI: $10.3892 /$ etm.2017.5357

\begin{abstract}
The present study aimed to determine the genotype and age distribution of high-risk human papillomavirus (HR-HPV) and evaluate HPV-DNA in subjects with abnormal cervical cytology results by using crowd-based cervical cancer screening cytology data. The Thinprep liquid-based cytologic test (TCT) was performed from January 2013 to January 2014 in the permanent residents of Liaocheng (China) aged 21-65 years who were married or had sexual intercourse. The number of screened women totaled 20,017, among whom 937 had abnormal results, 785 of which were recalled. For subjects in the age range of 21-65 years, an HR-HPV typing test using the fluorescence hybridization method. Among the 785 cases with abnormal TCT findings, repeated testing identified atypical squamous cells of unknown significance/atypical glandular cells in 478 , low-grade squamous intraepithelial lesions in 175, high squamous intraepithelial lesions in 127 and squamous cell carcinoma/adenocarcinoma in 5 cases. Among these types, infection rates of HR-HPV were 50.2, 77.1, 89.0 and $100 \%$, respectively. Of the 785 cases with abnormal TCT results, 493 (62.8\%) were HR-HPV-positive. A total of 16 types of HR-HPV were detected: HPV-16, -18, -31, -33, -35, $-39,-45,-51-53,-56,-58,-59,-66,68$ and 73 . Subjects infected with $\geq 2$ types were defined as having a multi-type infection. The infection rate was high in the age groups of 26-30 and 51-55 years, accounting for $87.7 \%(71 / 81)$ and $79.7 \%(51 / 64)$, respectively, while it was lower in the $>55$ years group at $28.6 \%$ (14/54). The top five types of HR-HPV (stated in a decreasing order regarding positivity rate) were HPV16 $(21.5 \%, 169 / 785)$, HPV52 (12.2\%, 96/785), HPV58 (9.8\%, 77/785), HPV33 (9.7\%, 76/785) and HPV18 (7.5\%, 59/785). Single-type infection was
\end{abstract}

Correspondence to: Dr Weizhi You, Department of Obstetrics and Gynecology, Liaocheng People's Hospital, 67 West Dongchang Road, Liaocheng, Shandong 252000, P.R. China

E-mail: weizhi_you268@126.com

Key words: epidemiology, human papillomavirus, liquid-based cytology test, uterine cervical neoplasms encountered in $45.0 \%(353 / 785)$ and multi-type infection in $17.8 \%$ (140/785), among which 98 cases had a two-type infection, 37 had a three-type infection, 2 had a four-type infection, 2 had a five-type infection and 1 case had a six-type infection. In the present study, differences in multi-type HR-HPV infection between groups with different TCT results were statistically significant. In conclusion, compared with CTC screening on its own, complementary HR-HPV testing is an effective method for screening for cervical cancer. The infection rate of HPV16, $-52,-58,-33$ and -18 was high among patients with cervical cytological abnormalities. Multi-type infection adds to the risk of malignancies. In Liaocheng, high-risk groups were aged 26-30 and 51-55. Attention should be paid during the screening and follow-up visits of these groups.

\section{Introduction}

Cervical cancer, the third most common malignant tumor type endangering women's health, has a high incidence rate in developing countries due to limitation of medical treatment for health conditions and lack of cervical cancer screening $(1,2)$. An annual estimate of 10,000 cases are newly diagnosed and 30,000 succumb to cervical cancer in China (3). With the development of detection methods, early-stage diagnosis, which has an important role in the prevention and treatment of cancer, has been successfully performed and been of benefit to the patients.

The implementation of the Pap smear test, a single method to screen for cervical cancer, has decreased the incidence rate of this malignancy. However, due to the low coverage, low dependence, high specificity subjective judgment and the discomfort of the patients, the method is not widely used in the clinic (4-7). The Thinprep liquid-based cytological test (TCT) provides the screening efficiency of cervical cytology with a better medium for cervical cells and avoidance of the disadvantages of the Pap smear test. However, cytological screening cannot be widely used for the prevention and treatment of cervical cancer, due to a series of weaknesses, such as limitation of morphological examination, tiredness of vision as well as low sensitivity and specificity $(8,9)$.

In the past decade, cervical cancer was evidenced to be closely correlated with HR-HPV infection (10). HPV 
testing has been approved to be a reasonable choice and to be widely used in the screening of cervical pre-cancerous diseases $(11,12)$. The risk of disease also depends on HPV types, along with the patient's genotype and environmental factors such as smoking (10). To date, >200 types of HPV have been recognized and it has been suggested that HR-HPV including types 16,18 , are able to induce cervical cancer, particularly in patients with chronic HR-HPV infection (13).

More than 50 years of experience in the US and European countries have revealed that cytological screening on its own or combined with HPV testing for feedback obviously decreased the incidence rate of cervical cancer (14). However, HPV testing during the first line of cervical cancer screening has remained controversial. HPV testing was reported to be more effective and sensitive than cytological methods and may replace cytology as the first-line screening of cervical cancer (15). However, HPV testing may also have high probability of ignoring cervical diseases that may be diagnosed by other methods of detection. The American Cancer Society, the American Society for Colposcopy and Cervical Pathology and the American Society of Clinical Pathology have proposed that the combination of cytology and HPV testing significantly increase the sensitivity in cervical cancer screens (16). In addition HR-HPV testing in subjects with abnormal TCT results may increase the sensitivity of cervical cancer detection and decrease the missed diagnosis rate (10).

Further studies suggested that the controversial role of HPV testing in the screening of cervical cancer may be due to differences in study populations. A meta-analysis revealed that subjects display significant inter-regional differences (17).

As a screening guide must take several factors and the real conditions of the district into consideration, the present study was performed. In 2008, Beijing commenced the screening of women for cervical and breast cancer free of charge and implemented this screening in the entire city in 2009 with good social feedback. Through this cervical cancer screening, the detection rate of cervical pre-cancerous lesions and early cervical cancer was significantly increased (18). Liaocheng People's Hospital (Lioacheng, China) in Shandong province began to screen women for cervical and breast cancer in January 2013; this service was provided free of charge in the first instance and once every two years thereafter. Until January 2014, nearly 20,000 married women residing in Liaocheng underwent cervical cancer screening. The present study focused on subjects with abnormal cervical cytology findings. HR-HPV testing was performed and an epidemiological analysis was conducted in order to effectively identify individuals with a high risk of cervical cancer. The present study may increase the screening efficacy, save resources and provide data for screening individuals with abnormal cytology results.

\section{Materials and methods}

Ethics statement. All of the testing procedures were in accordance with the ethical committee of Liaocheng People's Hospital and the international ethical guidelines for biomedical research involving humans (19). Patients provided informed written consent and agreed with their data being included in the present study.
Study design. According to the plan of Liaocheng (China) to screen women who were permanent residents for cervical and breast cancer, Liaocheng People's Hospital (Liaocheng, China) subjected 20,017 women to cervical cytology testing from January 2013 to January 2014, among which 937 cases had abnormal results. Abnormalities on liquid-based cytology are defined according to the Bethesda 2001 directive and include atypical squamous cell of unknown significance (ASCUS), atypical glandular cells of unknown significance (AGCUS), low-grade squamous intraepithelial lesions (LSIL), high-grade squamous intraepithelial lesion (HSIL), squamous cell carcinoma (SCC) and adenocarcinoma (ACA). In the present study, liquid-based cytology results of ASCUS or worse (ASCUS+) were considered abnormal (20). Patients with abnormal results were informed by trained staff via telephone communication, and were advised to undergo further treatment at the hospital. A file was generated for each of these patients and an information management platform was built in order to screen high-risk patients. A total of 785 patients were recalled for repeated CTC and HR-HPV-DNA testing. Their age ranged from 21 to 65 years with an average age of $36 \pm 9$ years and none of them were pregnant.

Cytology testing. The sampling of the secretion specimens was strictly performed during the non-menstrual period. No operation was performed within 3 days prior to sampling. For sampling, a cotton swab was used to obtain a secretion specimen from the cervix by wiping, and a cervical brush (ThinPrep 2000; Hologic, Inc., Beijing, China) was then applied to the cervical mouth and rotated clockwise for 4-5 circles. The cervical brush head was then put into a tube containing cell preservation liquid (PreservCyt ${ }^{\circledR}$ Solution; Hologic, Inc.). Tubes were kept in an upright position. Cervical liquid-based cytology tests were then performed by experienced cytology experts at the Department of Pathology of Liaocheng People's Hospital.

HPV DNA testing. Methods clinically used for HPV testing include cytology, immunohistochemistry, in situ hybridization, dot blot hybridization, nucleic acid blotting and polymerase chain reaction (PCR). In 2014, the US Food and Drug Administration (FDA) approved Cobas ${ }^{\circledR}$ HPV testing applied on its own in the first-line screening of women aged $\geq 25$ years (20). This method will decrease the status of cervical cytology in screening and become a method for diagnostically stratifying patients. At present, two major HR-HPV testing methods are used in China: The first one is the Hybrid Capture $2 \mathrm{HPV}$ test for quantitative testing of HR-HPV DNA, which is an established standard worldwide, and served as a comparator in the guidelines for accurate HPV testing methods (21-23). The second is a gene chip technology-based test, which combines PCR and reverse dot blot hybridization (24). The two methods are used to collect information about the probands' HPV infection status of the genital tract at different time-points, and they are widely used in initial screens for cervical diseases and follow-up visits.

In the present study, cervical cell samples from patients were kept for 2 weeks, within which one equal part of the samples of patients with abnormal cytology findings was transported to the central laboratory and stored at a temperature of $4^{\circ} \mathrm{C}$, 
Table I. Comparison between TCT results and positivity rate of high-risk human papillomavirus infection.

\begin{tabular}{lccccc}
\hline TCT typing & Total cases $(\mathrm{n})$ & Positive $(\mathrm{n})$ & Negative $(\mathrm{n})$ & Positive rate $(\%)$ & $\chi^{2}$ \\
\hline ASCUS/AGC & 478 & 240 & 238 & 50.2 & 88.1 \\
LSIL & 175 & 135 & 40 & 77.1 & 0.001 \\
HSIL & 127 & 113 & 14 & 89.0 \\
SCC/ACA & 5 & 5 & 0 & 100.0 \\
Total & 785 & 493 & 292 & 62.8 \\
\hline
\end{tabular}

TCT, Thinprep liquid-based cytological test; ASCUS, atypical squamous cells of undetermined significance; AGC, atypical glandular cells; LSIL, low-grade squamous intraepithelial lesion; HSIL, high-grade squamous intraepithelial lesion; SCC, squamous cell carcinoma; ACA, adenocarcinoma.

Table II. Comparison of infection rate [n, (\%)] of single- and multi-type high-risk human papillomavirus in subjects with different TCT results in Liaocheng.

\begin{tabular}{lcccc}
\hline TCT type & Cases tested $(\mathrm{n})$ & Single-type & Multi-type & $\chi^{2}$ \\
\hline ASCUS/AGC & 478 & $207(43.3)$ & $33(6.9)$ & 94.7 \\
LSIL & 175 & $101(57.7)$ & $34(19.4)^{\mathrm{a}}$ & $<0.01$ \\
HSIL & 127 & $45(35.4)$ & $68(53.5)^{\mathrm{a}, \mathrm{b}}$ & \\
SCC/ACA & 5 & $0(0.0)$ & $5(100.0)^{\mathrm{a}, \mathrm{b}}$ &
\end{tabular}

${ }^{\mathrm{a} P}<0.05$ compared with ASCUS/AGC group; ${ }^{\mathrm{b}} \mathrm{P}<0.05$ compared with LSIL. TCT, Thinprep liquid-based cytological test; ASCUS, atypical squamous cells of undetermined significance; AGC, atypical glandular cells; LSIL, low-grade squamous intraepithelial lesion; HSIL, high-grade squamous intraepithelial lesion; SCC, squamous cell carcinoma; ACA, adenocarcinoma.

where HPV-DNA testing was performed. Regular HPV testing included HPV-DNA sampling, DNA amplification, in situ hybridization detection and data processing with Luminex Data Collector version 1.7 (Luminex Corp., Austin, TX, USA). The procedure was performed according to the protocol of the Nucleic Acid Genotyping kit for Human Papillomavirus (cat. no. HPV27) provided by Shanghai Tellgen Life Science Co., Ltd. (Shanghai, China).

HPV genotype testing. Cervical cell samples were subjected to PCR for in vitro amplification of HPV DNA using the Nucleic Acid Genotyping kit for Human Papillomavirus. The Applied Biosystems ${ }^{\circledR} 7500$ Real-Time PCR System (Thermo Fisher Scientific, Waltham, MA, USA) was used as a platform, and according to the hybridization principle, DNA probes were hybridized on a low-density gene chip with attached nucleic acid probes. This experiment can test 27 HPV sub-types, which are divided into 17 high-risk and 10 low-risk HPV types (23). Through analysis of high-risk sub-type cases, HR-HPV includes HPV16, -18, -31, -33, -35, -39, -45, -51-53, -56, -58, -59, $-66,-26,-68$ and -73 . Positivity is defined as $\geq 103$ copies $/ \mathrm{ml}$ HR-HPV DNA. Infection with $\geq 2$ of the 17 types was defined as a multi-type infection. In the current study, differences in multi-type HR-HPV infection between groups with different TCT results were statistically significant.

Statistical analysis. SPSS 18.0 (SPSS, Inc., Chicago, IL, USA) was used for comparative and descriptive analysis. $\chi^{2}$ testing was used when comparing more than two groups, and data were compared in a line $\mathrm{x}$ column Table. $\mathrm{P}<0.05$ was considered to indicate a statistically significant difference.

\section{Results}

Cases with abnormal TCT results. Among the 785 cases with abnormal findings on TCT, repeated examination identified ASCUS/AGC in 478, LSIL in 175, HSIL in 127, and SCC/ACA in 5. In these groups, the infective rate of HR-HPV was 50.2, $77.1,89.0$ and $100 \%$, respectively. The HPV positivity rate was significantly associated with the degree of cervical cell abnormality according to the TCT $\left(\chi^{2}=88.1 ; \mathrm{P}<0.05\right.$; Table I).

Epidemiological distribution of HR-HPV. Of the 17 genotypes of HR-HPV covered by the testing kit, all were found in the screened subjects except HPV26. The infection rate of HR-HPV was $62.8 \%$ (493/785). Fig. 1 presents the 5 most frequently identified HR-HPV infection types, including HPV16 (21.5\%, 169/785), HPV52 (12.2\%, 96/785), HPV58 $(9.8 \%, 77 / 785), \operatorname{HPV} 33(9.7 \%, 76 / 785)$ and HPV18 $(7.5 \%$, 59/785). The HPV infection rate was high among cases of HSIL and cases of SCC and ACA. Single-type HR-HPV infection was encountered in $45.0 \%(353 / 785)$, while multi-type HR-HPV infection (including $\geq 2$ types) was found in $17.8 \%$ (140/785). There were 98 cases of two-type infection, 37 cases of three-type infection, 2 of four-type infection, 2 of five-type infection and 1 case of six-type infection. As presented in Fig. 1 and Table II, there were 478 cases of ASCUS and AGC, 207 of which possessed single-type infection (43.3\%) 
and 33 cases possessed two-type infection (6.9\%). A total of 175 cases of LSIL were identified, among which 101 cases had single-type infection (57.7\%) and 34 had multi-type infection (19.4\%; two- and three-type). There were 127 cases of HSIL, 45 of which had single-type HR-HPV infection and 68 had multi-type infection (53.5\%; two- and four-type infection). A total of 5 cases of SCC and ACA were identified, all of which had multi-type infection; among them, 2 cases were of three-type infection, 1 of four-type, 1 of five-type and 1 of six-type infection (Tables II and III, and Fig. 2).

Age distribution of HR-HPV-infected patients. As presented in Fig. 2, different age groups had a different infection rate of HPV. $\chi^{2}$ (line $\mathrm{x}$ column) testing revealed $\chi^{2}=377$ and the difference was statistically significant $(\mathrm{P}<0.05)$. Further comparison at $\alpha=0.0014$ demonstrated that the age groups with the highest infection rates were those of 26-30 and 51-55 years, while the infection rate was lowest in patients aged 56-60 and 61-65 years.

\section{Discussion}

Epidemiological and biological data have confirmed that continuous HR-HPV infection is the main cause of cervical cancer and its pre-cancerous lesions. There is a dispute with regard to whether HPV testing should be used as a primary screening method for cervical cancer. It is worth mentioning that cytology is not effective in the screening of cervical intraepithelial neoplasia grade 2+ (CIN2+) lesions. Analysis of European and North American cases indicated that the sensitivity rate is only $53 \%$, giving rise to a new era of molecular testing for HPV DNA (24). In May 2003, the US FDA approved the joint application of TCT and HPV-DNA HC2 testing for the primary screening of women aged $\geq 30$ years (17). In 2005, the World Health Organization's International Agency for Research on Cancer (IARC) recommended HR-HPV testing for use in the primary screening of cervical cancer (22). Stratified management of HR-HPV-positive patients is performed according to cytological testing results. Once women were tested as double negative, they are not tested for $\geq 5$ years, but a test is mandatory after 10 years (25); these guidelines are applied in Liaocheng People's Hospital. Cervical cancer screening guidelines by the American College of Obstetrics and Gynecology was published in 2014 proposed that HR-HPV testing is not recommended for women under the age of 30 years. The reason is that transient infections are common and even if they test positive, the infection will be cleared over a period of time. HPV infection may be transient and only a small number of patients infected will develop cancer after a long incubation period. As there are $>20$ sub-types (13), testing for single HPV types is not sufficient and a typing test is necessary. In recent years, the Chinese government has listed cervical cancer screening for women at appropriate ages as a major public health service, which is funded by the government. Therefore, the screening effect was evaluated in different regions, institutions and job positions, which may promote continuous improvement of cervical cancer screening work and ensure that the government's policy is implemented. The present study screened patients with abnormal TCT findings and subjected them to HR-HPV testing. It provided an evaluation

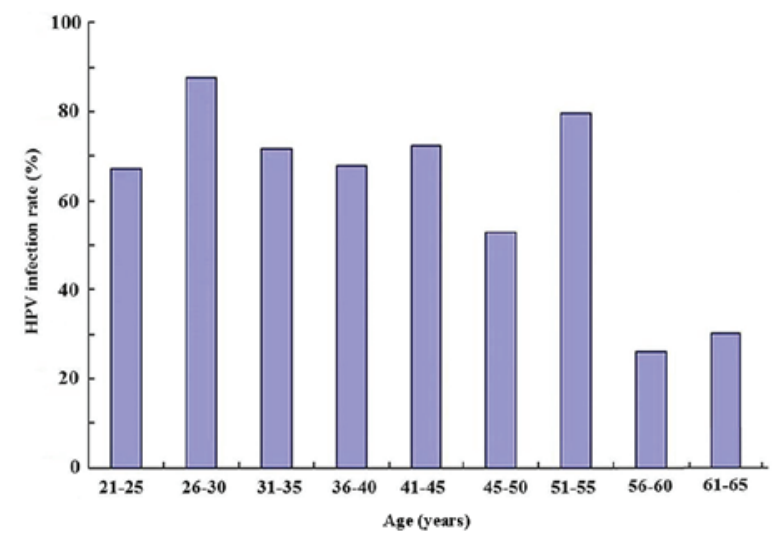

Figure 1. Rate of HPV infection in different age groups recalled for Thinprep liquid-based cytological testing. HPV, human papillomavirus.

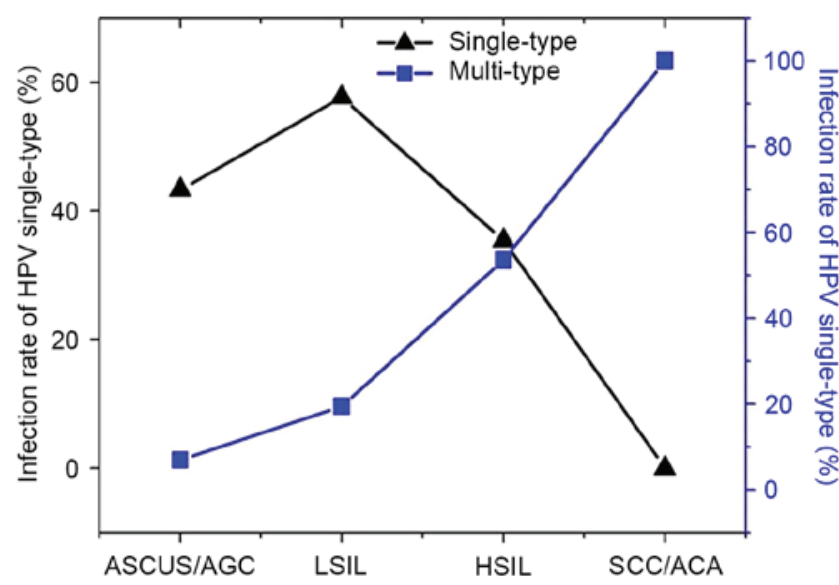

Figure 2. Comparison of infection rate of single- and multi-type high-risk-HPV in subjects with different Thinprep liquid-based cytological test results. HPV, human papillomavirus; ASCUS, atypical squamous cells of undetermined significance; AGC, atypical glandular cells; LSIL, low-grade squamous intraepithelial lesion; HSIL, high-grade squamous intraepithelial lesion; SCC, squamous cell carcinoma; ACA, adenocarcinoma.

of the epidemiological distribution of HPV in the local area and allows for comparison with previous studies. The age for testing was 21-65 years, with the lower limit of the age range being earlier than that reported in recent studies $(10,26)$. The purpose of the present study was to identify HR-HPV infection rates in patients with different abnormal TCT results as well as age distribution of infected patients and common genotypes of HPV in the local area through joint testing. The results may lead to the improvement of the effective screening rate of cervical lesions and ensure the future prevention and control of cervical cancer. In the follow-up study, patients whose cytology result was ASCUS were not further assessed in order to avoid unnecessary and intrusive inspection and decrease vaginal examination for HPV-negative patients. However, this did not reduce the discovery of CIN2+ and CIN3+ in patients with ASCUS (27). The diagnostic reliability is improved by eliminating suspicious ASCUS or low-grade lesions. Patients who had an increased risk of developing cervical cancer were differentiated from those with a low risk, which promoted the appropriate use of vaginal examination and pathological 
Table III. Epidemiological distribution of HR-HPV [positive rate in n (\%)] for subjects with different TCT results in Liaocheng.

\begin{tabular}{|c|c|c|c|c|c|}
\hline $\begin{array}{l}\text { HR-HPV } \\
\text { type }\end{array}$ & $\begin{array}{l}\text { Screened subjects } \\
\qquad(\mathrm{n}=785)\end{array}$ & $\begin{array}{c}\text { ASCUS/AGC } \\
(n=478)\end{array}$ & $\begin{array}{c}\text { LSIL } \\
(n=175)\end{array}$ & $\begin{array}{c}\text { HSIL } \\
(n=127)\end{array}$ & $\begin{array}{l}\text { SCC/ACA } \\
\quad(\mathrm{n}=5)\end{array}$ \\
\hline HPV16 & $169(21.5)$ & 76 (15.9) & $35(20)$ & $54(42.5)$ & $4(80)$ \\
\hline HPV18 & $59(7.5)$ & $21(4.4)$ & $19(10.9)$ & 15 (11.8) & $4(80)$ \\
\hline HPV31 & $36(4.6)$ & $15(3.1)$ & $6(3.4)$ & $14(11)$ & $1(20)$ \\
\hline HPV33 & $76(9.7)$ & $31(6.5)$ & $20(11.4)$ & $23(18.1)$ & $2(40)$ \\
\hline HPV35 & $2(0.3)$ & $2(0.4)$ & $0(0)$ & $0(0)$ & $0(0)$ \\
\hline HPV39 & $9(1.1)$ & $9(1.9)$ & $0(0)$ & $0(0)$ & $0(0)$ \\
\hline HPV45 & $2(0.3)$ & $1(0.2)$ & $1(0.6)$ & $0(0)$ & $0(0)$ \\
\hline HPV51 & $24(3.1)$ & 9 (1.9) & $3(1.7)$ & $12(9.4)$ & $0(0)$ \\
\hline HPV52 & $96(12.2)$ & $28(5.9)$ & $25(14.3)$ & $40(31.5)$ & $3(60)$ \\
\hline HPV53 & $23(2.9)$ & $14(2.9)$ & $6(3.4)$ & 2 (1.6) & $1(20)$ \\
\hline HPV56 & $51(6.5)$ & $20(4.2)$ & $15(8.6)$ & $15(11.8)$ & $1(20)$ \\
\hline HPV58 & 77 (9.8) & $24(5)$ & $22(12.6)$ & $29(22.8)$ & $2(40)$ \\
\hline HPV59 & $9(1.1)$ & $5(1)$ & $4(2.3)$ & $0(0)$ & $0(0)$ \\
\hline HPV66 & $18(2.3)$ & $6(1.3)$ & $9(5.1)$ & $2(1.6)$ & $1(20)$ \\
\hline HPV68 & $24(3.1)$ & $10(2.1)$ & $11(6.3)$ & 2 (1.6) & $1(20)$ \\
\hline HPV73 & $2(0.3)$ & $2(0.4)$ & $0(0)$ & $0(0)$ & $0(0)$ \\
\hline
\end{tabular}

Patients with abnormal TCT results included those with combined multiple HR-HPV infection. HR-HPV, high-risk human papillomavirus; TCT, Thinprep liquid-based cytological test; ASCUS, atypical squamous cells of undetermined significance; AGC, atypical glandular cells; LSIL, low-grade squamous intraepithelial lesion; HSIL, high-grade squamous intraepithelial lesion; SCC, squamous cell carcinoma; ACA, adenocarcinoma.

biopsy. In the follow-up study, screening time was relaxed for women negative on HPV testing.

Recent random comparisons among follow-up visits for HPV screening indicated that HPV DNA testing detects more high-level lesions than cytology (22). HPV-negative women have a low risk of developing CIN, and screening for them is performed at intervals of $\geq 5$ years (27). This largely reduces the screening cost as well as the psychological and economic burden of women screened. The present study found that HPV genotypes in different stages of cervical pre-cancerous lesions were significantly different. The infection rate of HR-HPV increased with the degree of cytological abnormality. This verified that HR-HPV infection is associated with the development of cervical lesions and it is the cause of cervical cancer. This result indicated that HR-HPV infection may predict high-level cervical lesions and high-degree abnormalities on cytology may suggest a high probability of HPV infection. Therefore, it is important for patients with abnormal cytological findings to be subjected to a HPV typing test. Individual evaluation may be performed in order to predict the risk of cervical lesions and determine a personal treatment plan. This is in consistent with the results of previous studies $(28,29)$.

In the present study, multi-type infection mostly comprised two-type infection, but hybrid infection of three, five or six types also occurred. Multi-type HR-HPV infection is more dangerous than single-type infection with regard to the generation of cervical cancer and HSIL, which has been demonstrated by previous studies $(30,31)$. In the present study, differences in multi-type HR-HPV infection between groups with different TCT results were statistically significant.
Although the impact of multi-type infection on the molecular mechanism and epidemiology of cervical cancer remains to be fully elucidated, follow-up visits for multi-type infection patients should be increased and further examination should be performed when necessary. The HPV genotype distribution has inter-regional differences and the 15 common sub-types reported by the IARC are HPV16, -18, -31, -33, -35, -39, -45, $-51,-52,-56,-58,-59,-66,-68$ and $-73(32)$.

The present study found that HPV16 was common in Liaocheng (China), followed by HPV52 and -58, while HPV18 was uncommon. However, HPV52 and -58 were associated with a lower incidence rate of cervical cancer than HPV18, indicating that HPV18 may have a greater carcinogenic effect or accelerate the development of cancer to a greater extent than the former types.

A recent study demonstrated that in a susceptible population, the 9vHPV vaccine prevented diseases associated with HPV31, $-33,-45,-52$ and -58 , led to the production of antibodies against HPV6, $-11,-16$ and -18 and did not perform worse than the qHPV vaccine (33). HPV typing revealed the genotype distribution in the region, which may provide scientific evidence for future screening and vaccine development.

A large-scale epidemiological survey identified age differences among HPV-infected individuals in different regions and populations (34). In line with this, the present study also found that the HR-HPV infection rate differs among different age groups. Among the 785 patients with abnormal TCT findings, the age groups of 26-30 and 51-55 years had the highest infection rates of 87.7 and $79.7 \%$, respectively. The infection rate was low women aged $>55$ at $28.6 \%$. The 
rate of HPV infection is high in women aged $>30$ years due to frequent sexual intercourse. However, their immune function is relatively strong, and most of the HPV infections are only transient and may be eliminated by the immune system. With increasing age, the function of the immune system decreases and the HPV infection rate increases. Changes of estrogen and progesterone levels in the peri-menopausal period are relatively large and is affect the metaplasia of the cervical epithelium. HPV replication may be supported by squamous epithelial metaplasia through its natural proliferation and differentiation process, thus increasing the HPV infection rate. Therefore, follow-up visits are important for women in the peri-menopausal period. A previous study demonstrated that with the polarization of cervical pre-cancerous lesions regarding the incidence age of cervical cancer, attention should be paid to women around the age of 30 years and those after the age of 50 years (35). Follow-up visits should be increased for HPV-positive patients. This has an important role in the prevention and treatment of cervical cancer.

In conclusion, the present study focused on the epidemiology of HPV infection in a population of patients with abnormal findings on cervical cytology in Liaocheng (China). According to the screening results, as compared with cytological screening on its own, HR-HPV typing is an effective screening method for cervical cancer. Identification of high-risk patients may strengthen the management of cervical cancer screening in the region in order to perform individualized treatment. Patients with abnormalities on cervical cytology in the region had a high infection rate of HPV16, -52, -58,-33 and -18. Multi-type infection was found to increase the risk of disease. In the region, high-risk individuals are mostly aged 26-30 and 51-55 years, and should therefore be subjected to stringent screening and follow-up visits. The genotype distribution in the region may provide scientific evidence for future screenings and vaccine development.

\section{References}

1. Zheng X, Liang P, Zheng Y, Yi P, Liu Q, Han J, Huang Y, Zhou Y, Guo J and Li L: Clinical significance of hTERC gene detection in exfoliated cervical epithelial cells for cervical lesions. Int J Gynecol Cancer 23: 758-790, 2013.

2. Schmeink CE, Bekkers RL, Massuger LF and Melchers WJ: The potential role of self-sampling for high-risk human papillomavirus detection in cervical cancer screening. Rev Med Virol 21: 139-153, 2011.

3. Zhao R, Zhang WY, Wu MH, Zhang SW, Pan J, Zhu L, Zhang YP, Li H, Gu YS and Liu XZ: Human papillomavirus infection in Beijing, People's Republic of China: A population-based study. Br J cancer 101: 1635-1640, 2009.

4. McPhail S, Elliss-Brookes L, Shelton J, Ives A, Greenslade M, Vernon S, Morris EJ and Richards M: Emergency presentation of cancer and short-term mortality. Br J Cancer 109: 2027-2034, 2013.

5. Jemal A, Ward E and Thun M: Declining death rates reflect progress against cancer. PLoS One 5: e9584, 2010.

6. Aggarwal P, Batra S, Gandhi G and Zutshi V: Comparison of Papanicolaou test with visual detection tests in screening for cervical cancer and developing the optimal strategy for low resource settings. Int J Gynecol Cancer 20: 862-868, 2010.

7. Wentzensen N and Silver MI: Biomarkers for cervical cancer prevention programs: The long and winding road from discovery to clinical use. J Low Genit Tract Dis 20: 191-194, 2016.

8. Belinson JL, Hu S, Niyazi M, Pretorius RG, Wang H, Wen C, Smith JS, Li J, Taddeo FJ, Burchette RJ and Qiao YL: Prevalence of type-specific human papillomavirus in endocervical, upper and lower vaginal, perineal and vaginal self-collected specimens: Implications for vaginal self-collection. Int J cancer 127 1151-1157, 2010.
9. Belinson JL, Pretorius RG, Enerson C, Garcia F, Cruz EP, Belinson SE, Yeverino García E and Brainard J: The Mexican Cervical Cancer Screening Trial (MECCS): Self-sampling for human papillomavirus with unaided visual inspection as a secondary screen. Int J Gynecol Cancer 19: 27-32, 2009.

10. Agorastos T, Chatzistamatiou K, Katsamagkas T, Koliopoulos G, Daponte A, Constantinidis T and Constantinidis TC; HERMES study group: Primary screening for cervical cancer based on high-risk human papillomavirus (HPV) detection and HPV 16 and HPV 18 genotyping, in comparison to cytology. PLoS One 10: e0119755, 2015.

11. Ronco G, Dillner J, Elfström KM, Tunesi S, Snijders PJ, Arbyn M, Kitchener H, Segnan N, Gilham C, Giorgi-Rossi P, et al: Efficacy of HPV-based screening for prevention of invasive cervical cancer: Follow-up of four European randomised controlled trials. Lancet 383: 524-532, 2014.

12. Cuzick J, Cadman L, Mesher D, Austin J, Ashdown-Barr L, Ho L, Terry G, Liddle S, Wright C, Lyons D and Szarewski A: Comparing the performance of six human papillomavirus tests in a screening population. Br J Cancer 108: 908-913, 2013.

13. Bouvard V, Baan R, Straif K, Grosse Y, Secretan B, El Ghissassi F, Benbrahim-Tallaa L, Guha N, Freeman C, Galichet L, et al: A review of human carcinogens-Part B: Biological agents. Lancet Oncol 10: 321-322, 2009.

14. Zhao C, Li Z, Nayar R, Levi AW, Winkler BA, Moriarty AT, Barkan GA, Rao J, Miller F, Fan F, et al: Prior high-risk human papillomavirus testing and Papanicolaou test results of 70 invasive cervical carcinomas dianosed in 2012: Results of a retrospective multicenter study. Arch Pathol Lab Med 139: 184-188, 2015.

15. Wright TC, Stoler MH, Behrens CM, Sharma A, Zhang G and Wright TL: Primary cervical cancer screening with human papillomavirus: End of study results from the ATHENA study using HPV as the first-line screening test. Gynecol Oncol 136: 189-197, 2015.

16. Saslow D, Solomon D, Lawson HW, Killackey M, Kulasingam SL, Cain J, Garcia FA, Moriarty AT, Waxman AG, Wilbur DC, et al: American Cancer Society, American Society for Colposcopy and Cervical Pathology, and American Society for Clinical Pathology screening guidelines for the prevention and early detection of cervical cancer. Am J Clin Pathol 137: 516-542, 2012.

17. Schiffman M, Boyle S, Raine-Bennett T, Katki HA, Gage JC, Wentzensen N, Kornegay JR, Apple R, Aldrich C, Erlich HA, et al: The role of human papillomavirus genotyping in cervical cancer screening: A large-scale evaluation of the cobas HPV test. Cancer Epidemiol Biomarkers Prev 24: 1304-1310, 2015.

18. Zhang YZ, Ma JF, Zhao FH, Xiang XE, Ma ZH, Shi YT, Hu SY and Qiao YL: Three-year follow-up results of visual inspection with acetic acid/Lugol's iodine (VIA/VILI) used as an alternative screening method for cervical cancer in rural areas. Chin J Cancer 29: 4-8, 2010 (In Chinese).

19. Council for International Organizations of Medical Sciences: International ethical guidelines for biomedical research involving human subjects. Bull Med Ethics: 17-23, 2002.

20. Solomon D, Davey D, Kurman R, Moriarty A, O'Connor D, Prey M, Raab S, Sherman M, Wilbur D, Wright T Jr, et al: The 2001 bethesda system: Terminology for reporting results of cervical cytology. JAMA 287: 2114-2119, 2002.

21. Stoler MH, Wright TC Jr, Sharma A, Apple R, Gutekunst K and Wright TL; ATHENA (Addressing THE Need for Advanced HPV Diagnostics) HPV Study Group: High-risk human papillomavirus testing in women with ASC-US cytology: Results from the ATHENA HPV study. Am J Clin Pathol 135: 468-475, 2011.

22. Cox JT, Castle PE, Behrens CM, Sharma A, Wright TC Jr and Cuzick J; Athena HPV Study Group: Comparison of cervical cancer screening strategies incorporating different combinations of cytology, HPV testing, and genotyping for HPV 16/18: Results from the ATHENA HPV study. Am J Obstet Gynecol 208: 184.e1-e184.e11, 2013.

23. Meijer CJ, Berkhof J, Castle PE, Hesselink AT, Franco EL, Ronco G, Arbyn M, Bosch FX, Cuzick J, Dillner J, et al: Guidelines for human papillomavirus DNA test requirements for primary cervical cancer screening in women 30 years and older. Int J Cancer 124: 516-520, 2009.

24. Söderlund-Strand A, Rymark P, Andersson P, Dillner J and Dillner L: Comparison between the Hybrid Capture II test and a PCR-based human papillomavirus detection method for diagnosis and posttreatment follow-up of cervical intraepithelial neoplasia. J Clin Microbiol 43: 3260-3266, 2005. 
25. Saslow D, Solomon D, Lawson HW, Killackey M, Kulasingam SL, Cain J, Garcia FA, Moriarty AT, Waxman AG, Wilbur DC, et al: American Cancer Society, American Society for Colposcopy and Cervical Pathology and American Society for Clinical Pathology screening guidelines for the prevention and early detection of cervical cancer. Am J Clin Pathol 137: 516-542, 2012.

26. Siddiqa A, Zainab M, Qadri I, Bhatti MF and Parish JL: Prevalence and genotyping of high risk human papillomavirus in cervical cancer samples from Punjab, Pakistan. Viruses 6: 2762-2777, 2014

27. Cuzick J, Arbyn M, Sankaranarayanan R, Tsu V, Ronco G, Mayrand MH, Dillner J and Meijer CJ: Overview of human papillomavirus-based and other novel options for cervical cancer screening in developed and developing countries. Vaccine 26 (Suppl 10): K29-K41, 2008.

28. Gage JC, Schiffman M, Katki HA, Castle PE, Fetterman B, Wentzensen N, Poitras NE, Lorey T, Cheung LC and Kinney WK: Reassurance against future risk of precancer and cancer conferred by a negative human papillomavirus test. J Natl Cancer Inst 106: pii: dju153, 2014.

29. Srodon M, Parry Dilworth H and Ronnett BM: Atypical squamous cells, cannot exclude high-grade squamous intraepithelial lesion: Diagnostic performance, human papillomavirus testing, and follow-up results. Cancer 108: 32-38, 2006.

30. Crowe E, Pandeya N, Brotherton JM, Dobson AJ, Kisely S, Lambert SB and Whiteman DC: Effectiveness of quadrivalent human papillomavirus vaccine for the prevention of cervical abnormalities: Case-control study nested within a population based screening programme in Australia. BMJ 348: g1458, 2014.
31. Massad LS, Einstein MH, Huh WK, Katki HA, Kinney WK, Schiffman M, Solomon D, Wentzensen N and Lawson HW; 2012 ASCCP Consensus Guidelines Conference: 2012 updated consensus guidelines for the management of abnormal cervical cancer screening tests and cancer precursors 121: 829-846, 2013.

32. Kitchener HC, Almonte M, Thomson C, Wheeler P, Sargent A, Stoykova B, Gilham C, Baysson H, Roberts C, Dowie R, et al: HPV testing in combination with liquid-based cytology in primary cervical screening (ARTISTIC): A randomised controlled trial. Lancet Oncol 10: 672-682, 2009.

33. Strander B, Hällgren J and Sparén P: Effect of ageing on cervical or vaginal cancer in Swedish women previously treated for cervical intraepithelial neoplasia grade 3: Population based cohort study of long term incidence and mortality. BMJ 348: f7361, 2014

34. Hu Y, Qian HZ, Sun J, Gao L, Yin L, Li X, Xiao D, Li D, Sun X, Ruan Y, et al: Anal human papillomavirus infection among HIV-infected and uninfected men who have sex with men in Beijing, China. J Acquir Immune Defic Syndr 64: 103-114, 2013. 35. Ogilvie GS, van Niekerk DJ, Krajden M, Martin RE, Ehlen TG Ceballos K, Peacock SJ, Smith LW, Kan L, Cook DA, et al: A randomized controlled trial of Human Papillomavirus (HPV) testing for cervical cancer screening: Trial design and preliminary results (HPV FOCAL Trial). BMC Cancer 10: 111, 2010. 